\title{
High-Fat/High-Sugar Diet Causes Metabolic Disorders and Cardiac Dysfunction: a New Model in Primates
}

\author{
Yan Qiu $^{1} \cdot$ Gui-e Xu ${ }^{1,2} \cdot$ Junjie Xiao ${ }^{1,2}$ (D) \\ Received: 23 February 2021 / Accepted: 28 February 2021 / Published online: 15 March 2021 \\ (C) The Author(s), under exclusive licence to Springer Science+Business Media, LLC, part of Springer Nature 2021
}

The number and proportion of aged people in the population is increasing. It was reported by World Health Organization (WHO) that the number of people aged 60 years or older was 1 billion in 2019 worldwide, and the number would grow to 1.4 billion by 2030 and 2.1 billion by 2050 . With the rapid development of society and great improvement of people's living standard, high-fat/high-sugar diet (HFSD) is common. High-fat/ high-sugar diet can lead to the development of obesity and metabolic diseases, including type 2 diabetes mellitus (T2DM), cardiovascular disease, and cognitive decline. However, few studies investigated the effects of HFSD on elderly people.

In a recent study, Zheng et al. identified novel changes in the metabolism, cardiac morphology, and cardiac function of aged Cynomolgus monkeys fed a HSFD [1]. The HFSD caused metabolic abnormalities and cardiac contractile dysfunction in aged monkeys. Although the detailed molecular mechanism for the HFSD effects on metabolic disorders and contractile dysfunction remains further investigation, this study provides a powerful model for the HFSD-induced metabolic and cardiac dysfunction.

Comment on: Shuang Zheng", Weijiang Tan", Xiang Li", Binglin Li, Baoyong Gong, W Glen Pyle, Jian Wu, Lei Li, Ting Luo, Yunzeng Zou, Feng Hua Yang ${ }^{*}$, Peter H Backx* . Aged monkeys fed a high-fat/ high-sugar diet recapitulate metabolic disorders and cardiac contractile dysfunction. J Cardiovasc Transl Res. 2021 Feb 16. doi: 10.1007/ s12265-021-10105-z.

Editor-in-Chief Enrique Lara-Pezzi oversaw the review of this article

Junjie Xiao

junjiexiao@shu.edu.cn

1 Institute of Geriatrics (Shanghai University), Affiliated Nantong Hospital of Shanghai University (The Sixth People's Hospital of Nantong), School of Medicine, Shanghai University,

Nantong 226011, China

2 Cardiac Regeneration and Ageing Lab, Institute of Cardiovascular Sciences, Shanghai Engineering Research Center of Organ Repair, School of Life Science, Shanghai University, 333 Nan Chen Road, Shanghai 200444, China
High fat and high sugar have now become the major constituent of modern diet. However, chronically high consumption of fat and sugar could lead to the occurrence of metabolic and cardiovascular disorders, which has been widely documented. Epidemiological study has highlighted the association between dietary patterns and cognitive decline in elders with average aged 69.3 years, and it is reported that western dietary pattern was associated with higher risk of cognitive decline [2]. Besides, chronic high-fat diet could induce myocardial hypertrophy and fibrosis. Obesity-related cardiac dysfunction epidemic heightened the need to identify a safe and effective therapy that can improve cardiac function and decrease the risk of cardiovascular disease. Large animal model provides a unique tool to study the mechanism of diet-induced cardiac dysfunction. Nonhuman primates (NHPs) are more similar to the human condition in terms of genetics, physiology, and metabolism.

The recent study established an aged monkey model with HFSD feeding, which is characterized with metabolic disorders and cardiac contractile dysfunction [1]. This is similar to that seen in the human condition. Serum metabolites analysis showed that HFSD significantly increased glucose and fatty acid levels. It is reported that the heart primarily consumes fatty acids, and the failing heart consumes more ketones and glutamate and releases more amino acids [3]. Echocardiography showed that the HFSD remodeled the heart and inhibited cardiac function in aged monkeys compared with regular diet (RD). To further explore the molecular signal changes caused by HFSD in aged myocardium, the authors investigated the inflammatory regulation and myofilament-associated phosphorylation signaling in the myocardium. It was showed that both the expression levels of inflammatory genes including IL-1 $\beta$, IL-6, IL-8, and IL-10 and the myofilament phosphorylation levels were significantly increased, suggesting activated inflammatory signaling and depressed myofilament contractility.

Taken together, the recent study used the aged Cynomolgus monkeys to model HFSD-induced metabolic and cardiac dysfunction, provides a powerful model for the 
human condition [1]. It would be interesting to know the role of specific metabolites playing in the progress of the agerelated cardio-metabolic disorders, or to identify new biomarkers for metabolic heart diseases in elderly. For example, what's the regulatory role of amino acids in heart failure? Whether and which amino acids can be used as biomarkers of age-related cardiovascular diseases? Further studies should combine the multi-omics, for example, RNAseq, and bioinformatics technology to investigate the molecular mechanism underlying the pathology.

Funding This work was supported by the grants from the National Natural Science Foundation of China (82020108002 and 81911540486 to JJ Xiao), the Innovation Program of Shanghai Municipal Education Commission (2017-01-07-00-09-E00042 to JJ Xiao), the grant from the Science and Technology Commission of Shanghai Municipality (20DZ2255400 and 18410722200 to JJ Xiao), and the "Dawn" Program of Shanghai Education Commission (19SG34 to JJ Xiao).

\section{Declarations}

Conflict of Interest The authors declare no competing interests.
Research Involving Human Participants and/or Animals This article does not contain any studies with human participants or animals performed by any of the authors.

Informed Consent This article does not contain any studies with human participants.

\section{References}

1. Zheng, S., et al. (2021). Aged monkeys fed a high-fat/high-sugar diet recapitulate metabolic disorders and cardiac contractile dysfunction. Journal of Cardiovascular Translational Research. https://doi.org/ 10.1007/s12265-021-10105-z

2. Tung, Y. T., et al. (2019). Physiological and biochemical effects of intrinsically high and low exercise capacities through multiomics approaches. Frontiers in Physiology, 10, 1201.

3. Murashige, D., et al. (2020). Comprehensive quantification of fuel use by the failing and nonfailing human heart. Science, 370(6514), 364-368.

Publisher's Note Springer Nature remains neutral with regard to jurisdictional claims in published maps and institutional affiliations. 